\title{
Assessment of mental health, knowledge, and attitude of patients with multiple sclerosis and neuromyelitis optica spectrum disorder in response to 2019 novel coronavirus
}

\author{
Vahid Shaygannejad ${ }^{1,2} \cdot$ Alireza Afshari-Safavi ${ }^{2,3} \cdot$ Boshra Hatef $^{4}$ (D) \\ Received: 31 August 2020 / Accepted: 12 November 2020 / Published online: 20 November 2020 \\ (C) Fondazione Società Italiana di Neurologia 2020
}

\begin{abstract}
Background With the recent pandemic of the novel coronavirus disease (COVID-19), multiple sclerosis (MS) and neuromyelitis optica spectrum disorder (NMOSD) patients for their compromised immune system have been in significant concern. Awareness and attitude about this virus have an important impact on infection prevention and coping with stress and anxiety. So we conducted this study to assess knowledge, attitude, and mental health status in MS and NMOSD patients within the COVID19 pandemic.

Method In this cross-sectional study, the link of Depression Anxiety Stress Scales-21 (DASS-21) and a self-administered structured questionnaire were sent through social media to MS and NMOSD patients and two control groups consisting of healthy and migraine individuals.

Results A total of 223 MS patients, 41 NMOSD, 29 migraine, and 245 healthy subjects participated in this study. MS patients had higher total DASS scores compared to healthy participants $(p=0.012)$. There were no significant differences among the study groups regarding knowledge and attitude. In MS patients, physical comorbidity was associated with a total score of attitude (OR $1.59,95 \%$ CI $0.53,2.66, p=0.004)$. We did not find association between other demographic and clinical variables with DASS scores, attitude, and knowledge in MS patients.

Conclusion The current data highlight the necessity of attitude, knowledge, and mental health assessment among MS and NMOSD patients. Further studies in other countries need to be carried to assess these points among MS and NMOSD patients.
\end{abstract}

Keywords Multiple sclerosis · Neuromyelitis optica spectrum disorder · Novel coronavirus disease (COVID-19) · Knowledge · Attitude $\cdot$ Mental health

\section{Introduction}

In late December 19, a novel coronavirus causing severe acute respiratory syndrome was identified in Wuhan, China [1]. Soon after, this virus has rapidly spread across all the world

Boshra Hatef

boshrahatef@bmsu.ac.ir

1 Department of Neurology, School of Medicine, Isfahan University of Medical Sciences, Isfahan, Iran

2 Isfahan Neurosciences Research Center, Isfahan University of Medical Sciences, Isfahan, Iran

3 Department of Biostatistics and Epidemiology, Faculty of Health, North Khorasan University of Medical Sciences, Bojnurd, Iran

4 Neuroscience Research Center, Baqiyatallah University of Medical Sciences, Vanak, Mollasadra, Tehran, Iran and emerges as a global public health threat. As of April 26, more than two million confirmed cases of coronavirus disease (COVID-19) had been identified in more than 210 countries or territories [2]. In early 2020, the first case of COVID-19 was confirmed in Iran. Up to now, a total of 88,194 confirmed cases and 5574 deaths have been identified [3].

Multiple sclerosis (MS) and neuromyelitis optica spectrum disorder (NMOSD) are both demyelinating diseases in the central nervous system. Some MS and all NMOSD patients require lifetime immunosuppressive therapy, which increases the risk of opportunistic infections [4]. It has been suggested that the prognosis of COVID-19 in patients who have received immunosuppressive agents may be different compared to those who have not utilized these therapies [5-9]. However, the effect of COVID-19 on MS and NMOSD patients is still unknown. 
As a result, the outbreak of COVID-19 for MS and NMOSD patients may be stressful and causes a feeling of anxiety, depression, and panic attack. There have been numerous studies to investigate the effect of psychological dysfunctions on the prognosis of these diseases. They indicated a robust association between mental health problems and reduced quality of life and severe disability [10-13]. A series of recent studies have indicated that COVID-19 has an association with mental health problems such as depression, anxiety, and stress in the general population and patients with chronic disorders [14-18]. However, few studies have investigated the mental health of MS and MS patients within COVID-19 pandemic [19].

Individuals' knowledge about COVID-19 has an association with attitude toward this disease and stress level [14]. Furthermore, misinformation and negative attitudes toward COVID-19 increase the risk of infection and are a barrier to COVID-19 control [20,21]. Several studies evaluated knowledge and attitude toward COVID-19 in health workers and the general population. However, perspectives and knowledge toward COVID-19 within the patients with MS and NMOSD remain unexplored.

Isfahan is one of the most infected provinces by nCOV-19 in Iran [22]. The high number of MS patients [23], along with the spread of COVID-19 outbreak in Isfahan and Iran, can be associated with a considerable risk of mortality and disability in MS and NMOSD patients [24-28]. Evaluation of mental health status, awareness, and attitude of MS and NMOSD patients toward COVID-19 are essential for the implementation of a specific and practical education program. So we conducted this study to assess the knowledge and attitude regarding new coronavirus disease among MS, NMOSD, and control individuals. We also survived depression, anxiety, and stress to evaluate mental health status among the study groups.

\section{Methods}

\section{Participants}

This cross-sectional study was conducted between March 8 and April 7, 2020. We checked our database (Isfahan Hakim MS database) to extract MS and NMSOD patients' information who had referred to the MS clinic, Kashani hospital, affiliated to Isfahan University of medical science. Our clinic covers the most considerable number of MS and NMOSD patients in Isfahan and the surrounding province. At the time point of diagnosis, all MS patients fulfilled the revised McDonald criteria of MS [29-32], and diagnosis of NMOSD was made based on the international consensus diagnostic criteria [33, 34].
To allow the comparison of mental health status, knowledge, and attitude regarding COVID-19 in MS and NMOSD patients with the general population, we recruited healthy individuals as control groups. Moreover, to compare these variables between MS and other patients who are not at an elevated risk, migraine patients were enrolled as second control groups. The reasons for selection of migraine as control group were as follows: (1) MS, NMOSD, and migraine usually affect population between the ages of 20 and 40 years; (2) these diseases are more prevalent in females, and (3) there has been no evidence regarding the elevated risk of COVID-19 in migraine [35].

We extracted MS, NMOSD, and migraine patients' contact information from our database or their medical documents. Contact information was used to send the questionnaires (see procedure section). We also extracted clinical information of MS patients. Clinical data included disease duration and disease severity score, which was measured using the Expanded Disability Status Scale (EDSS) [36]. The regional bioethics committee of the Isfahan University of Medical Sciences approved the study (IR.MUI.RESEARCH.REC.1398.830).

\section{Questionnaires}

We developed a self-administered questionnaire for data collection. This questionnaire is consisting of 4 parts that will be explained in turn.

- In the first section, demographic features were evaluated. It is consists of age, gender (male/female), education attainment, and marital status (single/married). In our country, education level is classified to a 4-point scale consisting elementary school ( $7-12$ years), middle school (12-14), high school (15-18), and university (19 years or more). We dichotomize the education level into "higher than diploma" and "diploma or lower than." The diploma or lower than includes elementary, middle, and high school degrees.

- In the second section, we evaluated the knowledge of participants regarding COVID-19 with ten questions. The incorrect answer and unanswered question (I do not know) were scored as 0 and a score of 1 given to the correct answers. A cumulative score of knowledge was measured for each participant, with higher scores indicating better knowledge about COVID-19.

- In the third section, we evaluated participants' attitudes toward COVID-19 with seven questions. Respondents are asked to rate these items using a four-item Likert-type scale from strongly agree to strongly disagree. We used two negative-attitude questions (Q1 and Q2) and five positive-attitude questions. Positive-attitude questions were scored as 1 for responses of strongly disagree, with cores increasing to 4 with responses of strongly agree and 
regarding negative-attitude questions, a score of 0 given to strongly agree, with cores increasing to 5 with responses of strongly disagree. A higher score indicates a better attitude regarding COVID-19.

- In the fourth section, we asked three multiple-choice questions. First, the participants were asked to choose the four most common symptoms of COVID-19 from a list of options. Next, they were asked about the main channel of receiving information about COVID-19. They could choose two of the following items: official media, social media platforms (such as WhatsApp, Telegram, Instagram, and Facebook), familial doctor, or recommendation was published by medical societies or other ways. In the end, we asked about major worries among participants about the corona. They could choose two of the following items: concern for myself due to the compromised immune system, worry for my close families, fear for elderly persons, and other concerns.

The face validity, content validity, internal consistency, and test-retest reliability of the instrument were measured to assess validity and reliability of the self-administered questionnaire. In quantitative face validity, all questions had an important score of $\geq 1.5$ and therefore remained in the questionnaire. After calculating the CVR and CVI coefficients, all questions had a CVR above 0.75 and a CVI above 0.75 . Cronbach's alpha was used to evaluate the internal consistency of the scales, and the results ranged from 0.445 to 0.675 . Also, the stability of the instruments were assessed by testretest reliability. Also, the stability of the instruments was assessed by test-retest reliability. The test-retest reliability measured by using Pearson's correlation coefficient was 0.797 and 0.818 for knowledge and attitude, respectively.

We also used the Iranian version of depression anxiety stress scale (DASS) to evaluate the emotional states of depression, anxiety, and stress [37, 38]. It consists of 21 items and seven items per each subscale. Scoring of the items is based on a 0 to 3 scale, and the total DASS score range was from 0 to 120 , with higher scores indicate higher severity of each domain.

\section{Procedure}

The main challenge faced by many researchers is the national quarantine. To resolve the problem, we used an online survey method for data collection. The online questionnaires were created using Google forms. We sent the link of the surveys through social media, including WhatsApp and Telegram, to individuals. The link of questionnaires was sent to 450 individuals, including $300 \mathrm{MS}$ patients, 76 NMOSD, and 64 migraines. The snowball sampling technique was used for gathering data from healthy population. We asked healthy individuals to recommend others to complete these questionnaires. By clicking the link, information about the study and the informed consent were brought up. After accepting to participate in the study, they filled up the questionnaires. In the end, they got auto the approval stage. After approving, they could not change their responses.

\section{Statistically analysis}

We presented descriptive data as mean (standard deviation [SD]) or frequency (\%) for interval and categorical variables, respectively. We used Chi-square test to compare sociodemographic data among the study groups. To compare the scores of knowledge, attitude, total, and subscales of DASS among the study groups, we conducted one-way ANOVA with Bonferroni correction.

Regression analysis was carried out to identify the influence of demographic and clinical variables on knowledge, attitude, and mental health status. The candidate variables included the following: age, sex (female or male), first EDSS score (EDSS at diagnosis date), current EDSS (EDSS at last visit), family history of MS (have or do not have), and physical and psychological comorbidity (have or do not have). The results of logistic regression analyses were recorded as odds ratio (OR), 95\% confidence interval $(\mathrm{CI})$, and $p$ value.

The level of significance was set at 0.05 (2-tailed). All statistical analysis procedures were performed using IBM SPSS Statistics (version 18; IBM Corporation, Armonk, NY, USA).

\section{Results}

A total of 223 MS patients, 41 NMOSD, 29 migraine, and 245 healthy individuals participated in the current study. No statistically significant differences among the study groups in demographic features, including age, sex, marital status, and educational level, were found. There was a statistically significant difference between MS and NMOSD regarding disease duration $(p=0.004)$. Table 1 summarized participants' demographic characteristics.

The results of knowledge and attitude assessment among the study groups are shown in Table 2 and Table 3.

Table 4 compares the mean scores for knowledge, attitude, and DASS among the study groups. We found statistically significant differences in scores of stress $(p=0.023)$ and total DASS $(p=0.045)$. Regarding stress score, a significant difference was found between MS and $\mathrm{HC}(p=0.011)$, as well as MS and NMOSD $(p=0.032)$. As regards to the total DASS score, we found a substantial difference between MS and HC $(p=0.012)$, but no difference between other groups was evident. 
Table 1 Participants' demographic characteristics

\begin{tabular}{|c|c|c|c|c|c|}
\hline Variables & $\operatorname{MS}(n=223)$ & $\operatorname{NMOSD}(n=41)$ & Migraine $(n=29)$ & Control $(n=245)$ & $p$ Value \\
\hline Sex; f, $n(\%)$ & $183(82.1 \%)$ & $32(78.0 \%)$ & $25(86.2 \%)$ & $185(75.5 \%)$ & 0.263 \\
\hline Age, mean (SD) & $35.88(7.49)$ & $34.09(9.51)$ & $35.96(727)$ & $34.20(7.48)$ & 0.084 \\
\hline Education; higher than diploma, $n(\%)$ & $158(70.9 \%)$ & $21(51.2 \%)$ & $20(69.0 \%)$ & $154(62.9 \%)$ & 0.059 \\
\hline Marriage; married, $n(\%)$ & $165(74.0 \%)$ & $30(73.2 \%)$ & $24(82.8 \%)$ & $162(66.1 \%)$ & 0.121 \\
\hline Disease duration, mean (SD) & $6.4(5.2)$ & $3.78(2.92)$ & NA & NA & 0.004 \\
\hline Fist EDSS; median (IQR) & $2(1)$ & NA & NA & NA & - \\
\hline Current EDSS; median (IQR) & $0(2)$ & NA & NA & NA & - \\
\hline MS familial; $\mathrm{y}, n(\%)$ & $36(20.7)$ & NA & NA & NA & - \\
\hline Physical comorbidity; y, $n(\%)$ & $64(36.4)$ & NA & NA & NA & - \\
\hline Psychological comorbidity; y, $n(\%)$ & $43(24.4)$ & NA & NA & NA & - \\
\hline
\end{tabular}

$f$ female, $y$ yes

Table 5 shows the response of the patients when asked about the symptoms of COVID-19. In all groups, the most common answers were as follows: dyspnea, fever, dry cough, and muscle pain. In NMOSD and HC, the main sources of COVID-19 information were social media. But most MS and migraine patients received their information through official media. In MS and NMOSD groups, most patients worried for themselves because of the compromised immune system. Approximately $45 \%$ of MS patients reported no worry regarding COVID-19. In both migraine and HC groups, most patients have not worried about COVID-19 for themselves but worry for family members.

On regression model, patients with somatic comorbidities have a better attitude compared to individuals without somatic comorbidities (OR 1.59, 95\%CI 0.53, 2.66, $p=0.004$ ). We did not find association between other demographic and clinical variables with DASS scores, attitude, and knowledge (Tables 5 and 6).

\section{Discussion}

In this study, we assessed mental health status, knowledge, and attitude toward COVID-19 among MS, NMOSD, migraine, and healthy individuals. The key findings were as follows: MS patients had higher total DASS score compared to healthy participants. Regarding knowledge and attitude, no substantial differences among the study groups were found. Another important finding was that the demographic and clinical characteristics of MS patients had no association with MS patients' knowledge, attitude, and the symptoms of psychological illness.

The COVID-19 pandemic is considered one of the greatest risks for public health in the recent century. Awareness regarding COVID-19 is indispensable for a concerned attitude and controlling and managing this disease. No substantial difference in knowledge and attitude regarding the new coronavirus disease and attitude among the study groups was found. Here, we discuss some specific results.

Social distancing is one of the most important ways to control the spread of COVID-19, particularly after society opening and for people who at higher risk for the disease. However, about $15 \%$ of both MS and NMOSD patients had poor knowledge about the role of social distancing in the prevention of disease spreading. Healthcare systems and clinicians should persuade patients to observe social distancing.

Another important finding was that more than three-fourths of all participants did not possess the requisite knowledge regarding the efficacy of antibiotics in the treatment of COVID-19. Previous studies showed an increased rate of arbitrarily antibiotic consumption and poor knowledge about antibiotics among Iranian population and some other countries [39-42]. The most common reasons for using antibiotics without prescription were fever, cough, and sore throat [43]. These symptoms are in constant with those that have been established in COVID-19 [44]. Following this reason, individuals with poor knowledge about antibiotic consumption are at higher risk for self-medication when they present the symptoms of COVID-19. It is strongly recommended that some educational programs should be conducted to improve knowledge of patients about the efficacy of antibiotic treatment in new coronavirus.

We also asked patients about the symptoms of COVID-19. Dyspnea, fever, dry cough, and muscle pain were reported as the most common symptoms of COVID-19 among all the study groups. This finding shows that our population has good awareness about the symptoms of COVID-19.

Overall, our MS and NMOSD samples have a good attitude concerning new coronavirus. It was found that $63 \%$ of MS, but none of NMOSD patients, believed that COVID-19 is impossible to be controlled. It may be that the high level of perceived stress in MS patients is a result of this poor attitude. 


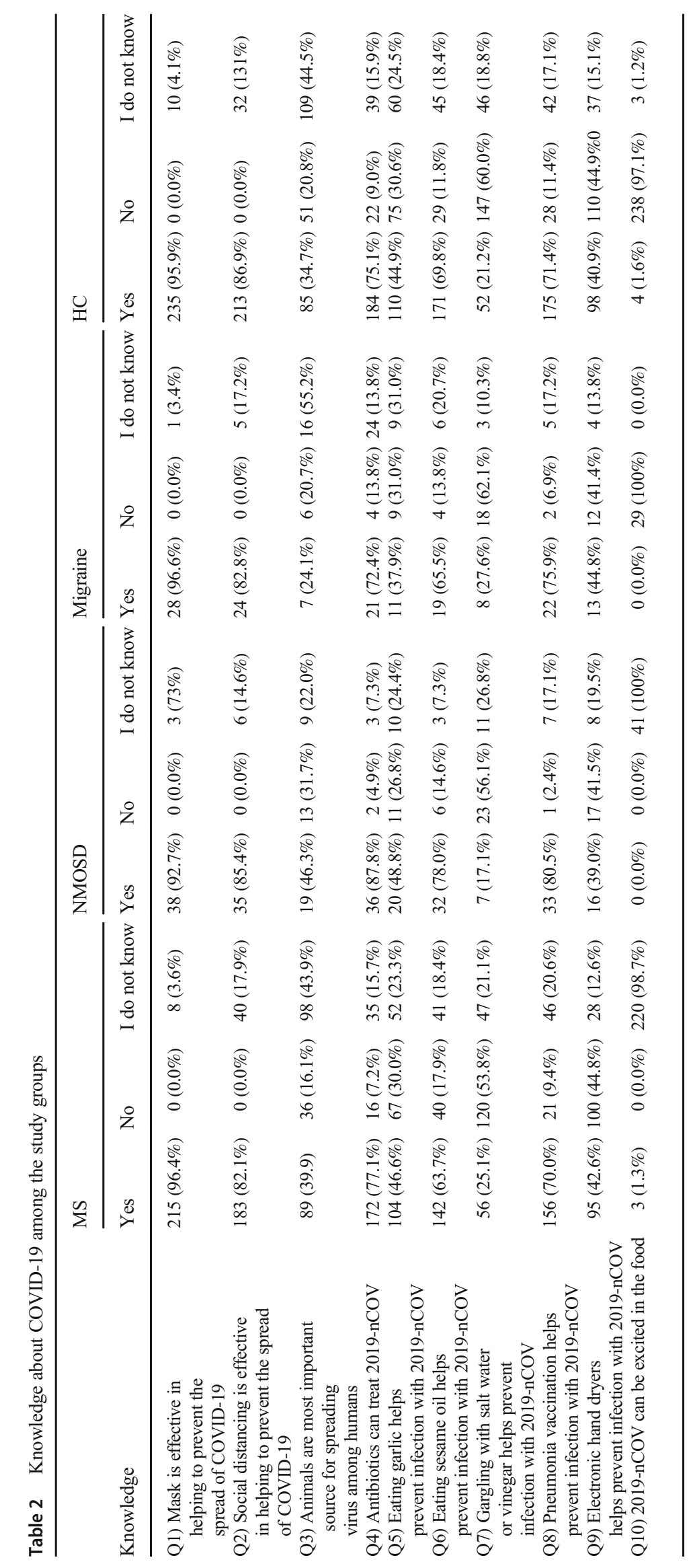




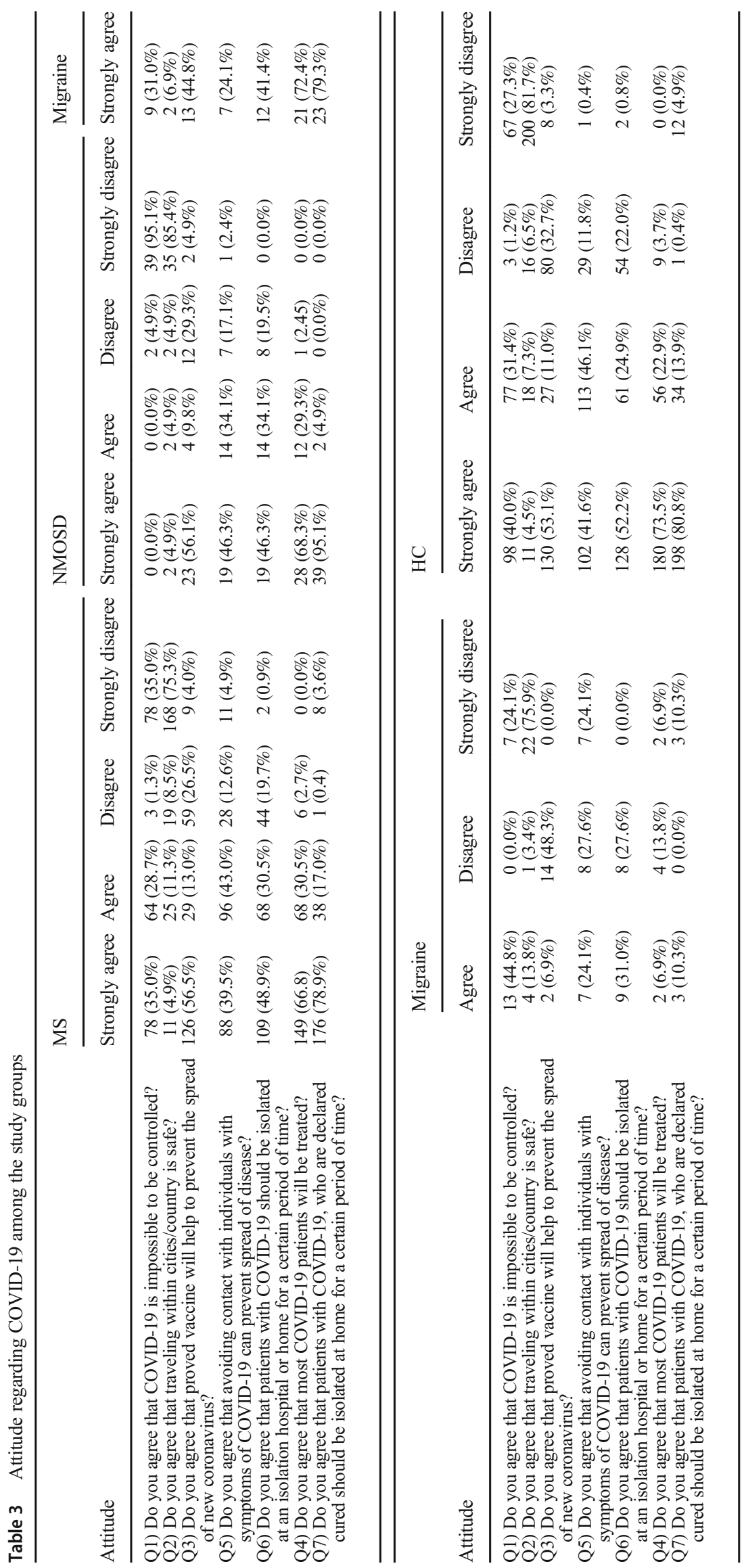


Table 4 Comparison of knowledge, attitude, and DASS score among the study groups

\begin{tabular}{llclll}
\hline & MS & NMOSD & Migraine & HC & $p$ Value \\
\hline Depression & $14.62(9.68)$ & $12.29(8.37)$ & $17.00(9.64)$ & $11.35(8.71)$ & 0.054 \\
Anxiety & $16.34(10.83)$ & $14.03(10.06)$ & $17.89(12.53)$ & $12.65(9.64)$ & 0.080 \\
Stress & $13.07(9.98)$ & $9.29(7.83)$ & $14.22(8.8)$ & $9.58(8.43)$ & 0.023 \\
Total DASS & $44.03(29.84)$ & $35.60(25.70)$ & $49.11(30.15)$ & $33.58(26.27)$ & 0.045 \\
Attitude & $24.6(2.0)$ & $24.87(1.84)$ & $24.10(2.22)$ & $24.57(2.15)$ & 0.450 \\
Knowledge & $6.80(1.58)$ & $6.23(1.78)$ & $7.57(1.39)$ & $6.32(1.50)$ & 0.107 \\
\hline
\end{tabular}

$P<0.05$ is considered statistically significant
Near $80 \%$ of both MS and NMOSD participants believed that avoiding contact with COVID-19 patients and isolation of these patients at home or hospital is necessary to control and management of the disease. In this study, about $90 \%$ of both MS and NMOSD patients agreed that most patients recovered. Almost all MS and NMOSD patients believed that cured COVID-19 patients might spread new coronavirus after recovery. While some researchers reported that some cured COVID-19 patients might be virus carriers [45], there remains a paucity of evidence on the spread of 2019-nCOV by patients after recovery.

The most common way of getting information about COVID-19 in MS and migraine patients was formal media.
A significant proportion of NMOSD and MS patients in the survey received their information via social media. With the COVID-19 outbreak, myths and fake news have rapidly spread on social media. Previous studies have emphasized the adverse effect of social media rumors and fabricated data on mental health, particularly on individuals who are venerable to the disease [46, 47]. Therefore, healthcare systems and governments provide authentic information and encourage people to avoid the spread of fake news.

In response to the 2019-nCOV pandemic, several recommendations from different local MS societies have been launched, which included practical advice for MS patients [48-51]. In this regard, the Iranian MS society published a
Table 5 Descriptive overview of the questions regarding most common symptoms of COVID19 , source of receiving information about COVID-19, and major worries about it

\begin{tabular}{lcccc}
\hline & $\begin{array}{l}\text { MS } \\
(n=223)\end{array}$ & $\begin{array}{l}\text { NMOSD } \\
(n=41)\end{array}$ & $\begin{array}{l}\text { Migraine } \\
(29)\end{array}$ & HC (n=245) \\
\hline Symptoms of COVID-19 & & & & \\
Dyspnea & $196(87.8 \%)$ & $34(82.9 \%)$ & $24(82.7 \%)$ & $178(72.6 \%)$ \\
Fever & $189(84.7 \%)$ & $31(75.6 \%)$ & $23(79.3 \%)$ & $176(71.8 \%)$ \\
Dry cough & $185(82.9 \%)$ & $28(68.2 \%)$ & $24(82.7 \%)$ & $163(66.5 \%)$ \\
Muscle pain & $107(47.9 \%)$ & $25(61.0 \%)$ & $17(58.6 \%)$ & $106(43.2 \%)$ \\
Headache & $89(39.9 \%)$ & $14(34.1 \%)$ & $16(55.1 \%)$ & $91(37.1 \%)$ \\
Rhinorrhea & $56(25.1 \%)$ & $12(29.2 \%)$ & $10(34.4 \%)$ & $50(20.4 \%)$ \\
Diarrhea & $54(24.2 \%)$ & $10(24.3 \%)$ & $8(27.5 \%)$ & $40(16.3 \%)$ \\
Dizziness & $31(13.9 \%)$ & $7(17.0 \%)$ & $6(20.6 \%)$ & $32(13.0 \%)$ \\
Vomit & $32(14.3 \%)$ & $8(19.5 \%)$ & $7(24.1 \%)$ & $38(15.5 \%)$ \\
Weight loss & $10(4.4 \%)$ & $1(2.4 \%)$ & $0(0.0 \%)$ & $3(1.2 \%)$ \\
Sources of COVID-19 information & & & & \\
Official media & $129(57.8 \%)$ & $22(53.6 \%)$ & $24(82.7 \%)$ & $112(45.7 \%)$ \\
Social media & $89(39.9 \%)$ & $24(58.5 \%)$ & $20(68.9 \%)$ & $125(51.0 \%)$ \\
Familial doctor & $65(29.1 \%)$ & $7(17.0 \%)$ & $10(34.4 \%)$ & $47(19.1 \%)$ \\
Others & $34(15.2 \%)$ & $9(21.9 \%)$ & $3(10.3 \%)$ & $27(11.0 \%)$ \\
Major worry regarding COVID-19 & $100(44.8 \%)$ & $8(19.5 \%)$ & $18(62.0 \%)$ & $27(11.20 \%)$ \\
Worry for myself due to the compromised & $133(55.1 \%)$ & $34(82.9 \%)$ & $5(17.2 \%)$ & $18(7.3 \%)$ \\
$\quad$ immune system & $19(8.5 \%)$ & $6(14.6 \%)$ & $6(20.6 \%)$ & $61(24.9 \%)$ \\
$\quad$ Worry for my close families & & & & \\
Worry for elderly persons & & & & \\
Others & & & & \\
\hline
\end{tabular}




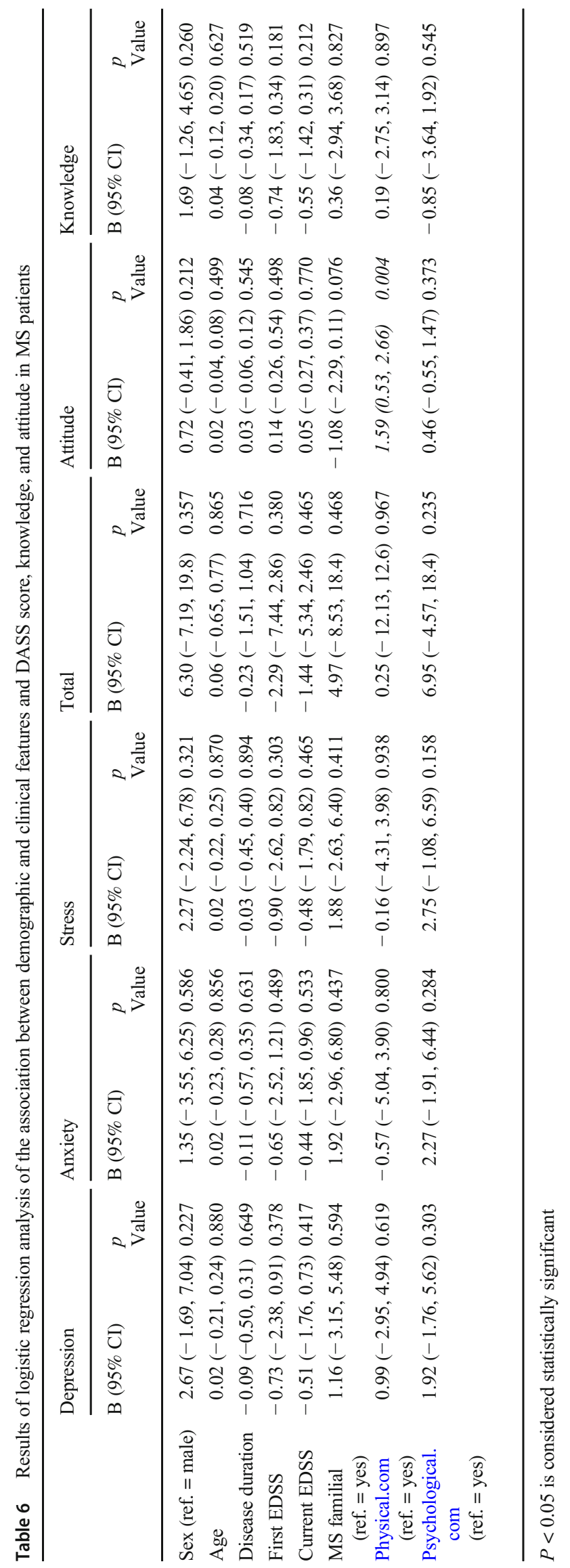


recommendation for MS patients [52]. These recommendations can be helpful in increasing the knowledge of patients about nCOV-2019. Surprisingly, only $30 \%$ of MS patients and $17 \%$ of NMOSD respondents used medical recommendation as a source for information. It is recommended that local MS societies take a more active role in providing health education to improve awareness about COVID-19 among their patients. They also should exert more effort to deliver the messages and recommendations to the patients.

As anticipated, the result showed a higher total DASS score in MS patients compared to healthy individuals. In accordance with our finding, a recent investigation on Indian population has shown emotional exhaust among more than two-thirds of respondents and emerges the importance of mental healthcare [14]. We suggested that mental health consultation should be adjunct to non-face-to-face MS and NMOSD evaluation and management care [53].

Recognizing the sources of stress is necessary in order to develop effective approaches. It has been reported that isolation, quarantine, social distancing, economic problems, and uncertainly about the future can affect mental health [47, 54]. Focusing on addressing specific concerns and fear among MS and NMOSD patients is also required. The current study found that MS and NMOSD patients were most worried about their health condition due to the compromised immune system. A much debated question is whether COVID-19 is associated with poor outcome in immunocompromised patients such as MS $[55,56]$. However, it is well-known that MS can increase infection-related healthcare utilization compared to the general population [57]. Future studies are therefore required in order to elucidate the association of MS and NMOSD with COVID-19.

Our results show that symptoms of psychological illness, awareness, and belief about COVID-19 had no association with clinical courses, disease severity, duration of disease, and psychological comorbidity. We only found an association between physical comorbidity and poor attitude. This finding suggests that the psychological effect of COVID-19 is independent of MS clinical features.

Our study limited in several ways. The major limitation of this study is the low reliability of the self-reported questionnaire. The nature of single-center study makes these findings less generalizable to the whole MS and NMOSD population. The study is also limited by the lack of information on the levels of depression, anxiety, and stress prior to COVID-19 outbreak. Therefore, it is not clear that the difference between MS and HC in DASS score is related either to the COVID-19 pandemic or MS burden [58]. A limited number of migraine patients who participated in this study are another limitation. Due to national quarantine, the face-to-face interview was impossible. Hence, we used an online survey method for gathering data. So, only individuals with a smart phone could participate in this study. Therefore, there is the possibility that respondents have a higher education level or better socioeconomic status compared to those who did not participate. Notwithstanding these limitations, as far as we know, this paper is the first to study the knowledge, attitude, and mental health status in MS and NMOSD patients within the COVID19 pandemic. The current data highlight the necessity of attitude, knowledge, and mental health assessment among MS and NMOSD patients. Further studies in other countries need to be carried to assess these points among MS and NMOSD patients.

\section{Compliance with ethical standards}

Conflict of interest The authors declare that they have no conflict of interest.

Ethical approval The study was approved in ethical board of Isfahan University of medical sciences.

Informed consent Written Informed consent was obtained from all of the participants.

\section{References}

1. Huang C, Wang Y, Li X, Ren L, Zhao J, Hu Y, Zhang L, Fan G, Xu J, Gu X, Cheng Z, Yu T, Xia J, Wei Y, Wu W, Xie X, Yin W, Li H, Liu M, Xiao Y, Gao H, Guo L, Xie J, Wang G, Jiang R, Gao Z, Jin Q, Wang J, Cao B (2020) Clinical features of patients infected with 2019 novel coronavirus in Wuhan, China. Lancet 395(10223):497506

2. World Health Organization (2020) Coronavirus disease 2019 (COVID-19) situation report -109. May 8, 2020. https://www. who.int/emergencies/diseases/novel-coronavirus-2019/situationreports. Accessed 9 May 2020

3. World Health Organization (2020) Coronavirus disease (COVID2019) situation report -108. https://www.who.int/emergencies/ diseases/novel-coronavirus-2019/situation-reports. Accessed 7 May 2020

4. Epstein DJ, Dunn J, Deresinski S (2018) Infectious complications of multiple sclerosis therapies: implications for screening, prophylaxis, and management. Open Forum Infect Dis: Oxford University Press US 5:ofy 174

5. Zhu L, Xu X, Ma K, Yang J, Guan H, Chen S, Chen Z, Chen G (2020) Successful recovery of COVID-19 pneumonia in a renal transplant recipient with long-term immunosuppression. Am J Transplant 20:1859-1863

6. Ghajarzadeh M, Bonavita S (2020) Are patients with multiple sclerosis (MS) at higher risk of COVID-19 infection? Neurol Sci 41(9): 2315-2316

7. Dalla Costa G, Leocani L, Montalban X, Guerrero AI, Sørensen PS, Magyari M et al (2020) Real-time assessment of COVID-19 prevalence among multiple sclerosis patients: a multicenter European study. Neurol Sci 41(7):1647-1650

8. Louapre C, Collongues N, Stankoff B, Giannesini C, Papeix C, Bensa C, Deschamps R, Créange A, Wahab A, Pelletier J, Heinzlef O, Labauge P, Guilloton L, Ahle G, Goudot M, Bigaut K, Laplaud DA, Vukusic S, Lubetzki C, de Sèze J, for the Covisep investigators (2020) Clinical characteristics and outcomes in patients with coronavirus disease 2019 and multiple sclerosis. JAMA Neurol 77(9):1079-1088 
9. Devogelaere J, D'hooghe MB, Vanderhauwaert F, D'haeseleer M (2020) Coronavirus disease 2019: favorable outcome in an immunosuppressed patient with multiple sclerosis. Neurol Sci 41(8): 1981-1983

10. Beiske A, Svensson E, Sandanger I, Czujko B, Pedersen E, Aarseth $\mathrm{J}$ et al (2008) Depression and anxiety amongst multiple sclerosis patients. Eur J Neurol 15(3):239-245

11. Buljevac D, Hop W, Reedeker W, Janssens A, Van der Meche F, Van Doorn P et al (2003) Self reported stressful life events and exacerbations in multiple sclerosis: prospective study. Bmj. 327(7416):646-640

12. Brown R, Tennant C, Sharrock M, Hodgkinson S, Dunn S, Pollard J (2006) Relationship between stress and relapse in multiple sclerosis: part II. Direct and indirect relationships. Mult Scler J 12(4): 465-475

13. Chanson JB, Zéphir H, Collongues N, Outteryck O, Blanc F, Fleury M, Vermersch P, de Seze J (2011) Evaluation of health-related quality of life, fatigue and depression in neuromyelitis optica. Eur J Neurol 18(6):836-841

14. Roy D, Tripathy S, Kar SK, Sharma N, Verma SK, Kaushal V (2020) Study of knowledge, attitude, anxiety \& perceived mental healthcare need in Indian population during COVID-19 pandemic. Asian J Psychiatr 51:102083

15. Wu W, Zhang Y, Wang P, Zhang L, Wang G, Lei G, Xiao Q, Cao X, Bian Y, Xie S, Huang F, Luo N, Zhang J, Luo M (2020) Psychological stress of medical staffs during outbreak of COVID19 and adjustment strategy. J Med Virol 92:1962-1970. https://doi. org/10.1002/jmv. 25914

16. Moghadasi N (2020) Evaluation of the level of anxiety among Iranian multiple sclerosis fellowships during the outbreak of COVID-19. Arch Iran Med 23(4):283

17. Shanafelt T, Ripp J, Trockel M (2020) Understanding and addressing sources of anxiety among health care professionals during the COVID-19 pandemic. Jama. 323:2133-2134

18. Orsini A, Corsi M, Santangelo A et al (2020) Challenges and management of neurological and psychiatric manifestations in SARSCoV-2 (COVID-19) patients. Neurol Sci 41(9):2353-2366. https:// doi.org/10.1007/s10072-020-04544-w

19. Naser Moghadasi A (2020) One aspect of coronavirus disease (COVID-19) outbreak in Iran: high anxiety among MS patients. Mult Scler Relat Disord 41:102138. https://doi.org/10.1016/j. msard.2020.102138

20. Srichan P, Apidechkul T, Tamornpark R, Yeemard F, Khunthason S, Kitchanapaiboon S, Wongnuch P, Wongphaet A, Upala P, (2020) Knowledge, attitude and preparedness to respond to the 2019 novel coronavirus (COVID-19) among the bordered population of northern Thailand in the early period of the outbreak: a cross-sectional study. Available at SSRN: https://ssrn.com/ abstract $=3546046$ or https://doi.org/10.2139/ssrn.3546046

21. Zhang M, Zhou M, Tang F, Wang Y, Nie H, Zhang L, You G (2020) Knowledge, attitude, and practice regarding COVID-19 among healthcare workers in Henan, China. J Hosp Infect 105(2): 183-187. https://doi.org/10.1016/j.jhin.2020.04.012

22. Javanmard SH, Nasirian M, Ataei B, Vaseghi G, Vaezi A, Changiz $\mathrm{T}$ (2020) Isfahan COvid-19 REgistry (I-CORE): design and methodology. J Res Med Sci 25(1):32

23. Azami M, YektaKooshali MH, Shohani M, Khorshidi A, Mahmudi L (2019) Epidemiology of multiple sclerosis in Iran: a systematic review and meta-analysis. PLoS One 14(4):e0214738. https://doi. org/10.1371/journal.pone.0214738

24. Sahraian MA, Azimi A, Navardi S, Ala S, Moghadasi AN (2020) Evaluation of the rate of COVID-19 infection, hospitalization and death among Iranian patients with multiple sclerosis. Mult Scler Relat Disord 46:102472

25. Safavi F, Nourbakhsh B, Azimi AR (2020) B-cell depleting therapies may affect susceptibility to acute respiratory illness among patients with multiple sclerosis during the early COVID-19 epidemic in Iran. Mult Scler Relat Disord 43:102195

26. Barzegar M, Mirmosayyeb O, Ghajarzadeh M, Nehzat N, Vaheb S, Shaygannejad V, Vosoughi R (2020) Characteristics of COVID-19 disease in multiple sclerosis patients. Mult Scler Relat Disord 45: 102276

27. Sahraian MA, Azimi A, Navardi S, Rezaeimanesh N, Naser Moghadasi A (2020) Evaluation of COVID-19 infection in patients with neuromyelitis optica spectrum disorder (NMOSD): a report from Iran. Mult Scler Relat Disord 44:102245. https://doi.org/10. 1016/j.msard.2020.102245

28. Mirmosayyeb O, Vaheb S, Barzegar M, Shaygannejad V, Bonavita S, Ghajarzadeh M (2020) Screening neuromyelitis optica patients for COVID-19 infection. Autoimmun Rev 19:102669

29. McDonald WI, Compston A, Edan G, Goodkin D, Hartung HP, Lublin FD et al (2001) Recommended diagnostic criteria for multiple sclerosis: guidelines from the international panel on the diagnosis of multiple sclerosis. Ann Neurol 50(1):121-127

30. Polman CH, Reingold SC, Edan G, Filippi M, Hartung HP, Kappos L, Lublin FD, Metz LM, McFarland HF, O'Connor PW, SandbergWollheim M, Thompson AJ, Weinshenker BG, Wolinsky JS (2005) Diagnostic criteria for multiple sclerosis: 2005 revisions to the "McDonald criteria". Ann Neurol 58(6):840-846

31. Polman CH, Reingold SC, Banwell B, Clanet M, Cohen JA, Filippi M, Fujihara K, Havrdova E, Hutchinson M, Kappos L, Lublin FD, Montalban X, O'Connor P, Sandberg-Wollheim M, Thompson AJ, Waubant E, Weinshenker B, Wolinsky JS (2011) Diagnostic criteria for multiple sclerosis: 2010 revisions to the McDonald criteria. Ann Neurol 69(2):292-302

32. Thompson AJ, Banwell BL, Barkhof F, Carroll WM, Coetzee T, Comi G, Correale J, Fazekas F, Filippi M, Freedman MS, Fujihara K, Galetta SL, Hartung HP, Kappos L, Lublin FD, Marrie RA, Miller AE, Miller DH, Montalban X, Mowry EM, Sorensen PS, Tintoré M, Traboulsee AL, Trojano M, Uitdehaag BMJ, Vukusic S, Waubant E, Weinshenker BG, Reingold SC, Cohen JA (2018) Diagnosis of multiple sclerosis: 2017 revisions of the McDonald criteria. Lancet Neurol 17(2):162-173

33. Wingerchuk DM, Lennon VA, Pittock SJ, Lucchinetti CF, Weinshenker BG (2006) Revised diagnostic criteria for neuromyelitis optica. Neurology. 66(10):1485-1489

34. Wingerchuk DM, Banwell B, Bennett JL, Cabre P, Carroll W, Chitnis T, de Seze J, Fujihara K, Greenberg B, Jacob A, Jarius S, Lana-Peixoto M, Levy M, Simon JH, Tenembaum S, Traboulsee AL, Waters P, Wellik KE, Weinshenker BG, International Panel for NMO Diagnosis (2015) International consensus diagnostic criteria for neuromyelitis optica spectrum disorders. Neurology. 85(2): 177-189

35. Parodi IC, Poeta MG, Assini A, Schirinzi E, Del Sette P (2020) Impact of quarantine due to COVID infection on migraine: a survey in Genova, Italy. Neurol Sci 41(8):2025-2027

36. Kurtzke JF (1983) Rating neurologic impairment in multiple sclerosis: an expanded disability status scale (EDSS). Neurology 33(11):1444

37. Henry JD, Crawford JR (2005) The short-form version of the depression anxiety stress scales (DASS-21): construct validity and normative data in a large non-clinical sample. Br J Clin Psychol 44(2):227-239

38. Asghari A, Saed F, Dibajnia P (2008) Psychometric properties of the depression anxiety stress scales-21 (DASS-21) in a non-clinical Iranian sample. Int J Psychol 2(2):82-102

39. Abbasian H, Hajimolaali M, Yektadoost A, Zartab S (2019) Antibiotic utilization in Iran 2000-2016: pattern analysis and benchmarking with organization for economic co-operation and development countries. J Res Pharm Pract 8(3):162-167

40. Jamhour A, El-Kheir A, Salameh P, Hanna PA, Mansour H (2017) Antibiotic knowledge and self-medication practices in a developing 
country: a cross-sectional study. Am J Infect Control 45(4):384388

41. Enato EF, Sounyo AA, Einarson TR (2011) Medication utilization and illness management study in Nigeria. Ann Pharmacother 45(78):924-930

42. Torres N, Chibi B, Middleton L, Solomon V, MashambaThompson T (2019) Evidence of factors influencing selfmedication with antibiotics in low and middle-income countries: a systematic scoping review. Public Health 168:92-101

43. Hosseinzadeh K, Azimian J (2017) Iranians' self-report knowledge and practice about arbitrary use of antibiotics. J Clin Diagn Res 11(8):FC06-FC09

44. Borges do Nascimento IJ, Cacic N, Abdulazeem HM, von Groote TC, Jayarajah U, Weerasekara I, Esfahani MA, Civile VT, Marusic A, Jeroncic A, Carvas Junior N, Pericic TP, Zakarija-Grkovic I, Meirelles Guimarães SM, Luigi Bragazzi N, Bjorklund M, SofiMahmudi A, Altujjar M, Tian M, Arcani DMC, O'Mathúna DP, Marcolino MS (2020) Novel coronavirus infection (COVID-19) in humans: a scoping review and meta-analysis. J Clin Med 9(4):941. https://doi.org/10.3390/jcm9040941

45. Lan L, Xu D, Ye G, Xia C, Wang S, Li Y, Xu H (2020) Positive RT-PCR test results in patients recovered from COVID-19. Jama. 323:1502-1503

46. Everts J (2013) Announcing swine flu and the interpretation of pandemic anxiety. Antipode. 45(4):809-825

47. Banerjee D (2020) The COVID-19 outbreak: crucial role the psychiatrists can play. Asian J Psychiatr 50:102014

48. National MS society (2020) What You need to know about coronavirus (COVID-19). https://www.nationalmssociety.org/Whatyou-need-to-know-about-Coronavirus-(COVID-19). Accessed 15 Apr 2020

49. United Kingdom MS society (2020) COVID-19 coronavirus and MS treatments. https:/www.mssociety.org.uk/about-ms/ treatments-and-therapies/disease-modifying-therapies/covid-19coronavirus-and-ms. Accessed 15 Apr 2020

50. Ireland MS society (2020) COVID-19 and MS. https://www.mssociety.ie/covid-19-and-ms (accessed April 15, 2020)

51. Australia MS society (2020) COVID-19 information for people with MS. https://www.ms.org.au/coronavirus. Accessed 8 Apr 2020

52. Iran MS society (2020) Everything MS Patients should know about COVID-19. http://www.iranms.ir/fa. Accessed 22 Apr 2020

53. Cohen BH, Busis NA, Ciccarelli L (2020) Coding in the world of COVID-19: non-face-to-face evaluation and management care. Continuum. 26(3):1-25

54. Brooks SK, Webster RK, Smith LE, Woodland L, Wessely S, Greenberg N, Rubin GJ (2020) The psychological impact of quarantine and how to reduce it: rapid review of the evidence. Lancet 395:912-920

55. Multiple sclerosis and related disorders (2020) Rethinking high-risk groups in COVID-19. Article in press. https://www.msard-journal. com/article/S2211-0348(20)30215-7/fulltext. Accessed 23 Apr 2020

56. D'Antiga L (2020) Coronaviruses and immunosuppressed patients. The facts during the third epidemic. Liver Transpl 26:832-834

57. Wijnands JM, Kingwell E, Zhu F, Zhao Y, Fisk JD, Evans C et al (2017) Infection-related health care utilization among people with and without multiple sclerosis. Mult Scler J 23(11):1506-1516

58. Corallo F, Buono VL, Genovese R, Palmeri R, Di Cara M, Rifici C et al (2019) A complex relation between depression and multiple sclerosis: a descriptive review. Neurol Sci 40(8):1551-1558

Publisher's note Springer Nature remains neutral with regard to jurisdictional claims in published maps and institutional affiliations. 